Supporting Information for:

\title{
Interfacial States, Energetics, and Atmospheric Stability of Large-Grain Antifluorite $\mathrm{Cs}_{2} \mathrm{TiBr}_{6}$
}

\author{
Jocelyn L. Mendes, ${ }^{\dagger}$ Weiran Gao, ${ }^{\dagger}$ Julia L. Martin, ${ }^{\dagger}$ Alexander D. Carl, ${ }^{\dagger}$ \\ N. Aaron Deskins, ${ }^{\ddagger}$ Sergio Granados-Focil, ${ }^{\mathbb{T}}$ and Ronald L. Grimm ${ }^{*, \dagger}$ \\ tDepartment of Chemistry and Biochemistry; Life Science and Bioengineering Center; \\ Worcester Polytechnic Institute, 100 Institute Road, Worcester, MA 01609, United States \\ $\ddagger$ Department of Chemical Engineering; \\ Worcester Polytechnic Institute, 100 Institute Road, Worcester, MA 01609, United States \\ IIGustaf H. Carlson School of Chemistry and Biochemistry; \\ Clark University, Worcester, Massachusetts 01610, United States \\ E-mail: grimm@wpi.edu,0000-0003-0407-937X
}




\section{S1. EXPERIMENTAL SECTION}

\section{S1.1. Materials and Chemicals}

All chemicals were used as received unless otherwise noted. Chemicals used in the synthesis of cesium titanium bromide included cesium bromide $(\mathrm{CsBr}, 99.9 \%$ trace metals basis, Sigma Aldrich), and titanium(IV) bromide ( $\mathrm{TiBr}_{4}, 98 \%$, Sigma Aldrich) that was further purified by sublimation. Dichloromethane (DCM, ACS Grade, Fisher) was dried in a commercial solvent drying system and was further degassed then stored over molecular sieves ( $3 \AA$, Alfa Aesar, freshly activated prior to use). A Milli-Q filtration system provided all water at $18 \mathrm{M} \Omega \mathrm{cm}$ resistivity (Millipore).

Inert environments for sample synthesis, processing, reactions, and storage included an argon-purged (ultrahigh purity, UHP, Airgas) Schlenk line equipped with an oil diffusion pump having a base pressure below $1 \times 10^{-3}$ torr. Larger inert environments included a nitrogen-purged (UHP, Airgas) recirculating glove box (mBraun Inc., Stratham, $\mathrm{NH}$ ) with an active catalyst to maintain oxygen concentrations below $1 \mathrm{ppm}$, as measured by a commercial $\mathrm{O}_{2}$ sensor. A commercial $\mathrm{N}_{2}$ generator (Model N2-45, Parker Balston, Lancaster, NY) provided the atmosphere for a flush box (Terra Universal, Fullerton, CA) with an $\mathrm{O}_{2}$ concentration below $5 \mathrm{ppt}$ (thousand), as measured at a commercial $\mathrm{O}_{2}$ sensor.

\section{S1.2. Synthesis of Large-Grain $\mathrm{Cs}_{2} \mathrm{TiBr}_{6}$}

Prior to the synthesis of cesium titanium bromide, titanium(IV) bromide was purified via vacuum sublimation on the Schlenk line and transferred to the recirculating glove box. Cesium bromide was opened under an air ambient, covered with a laboratory wipe, and remained in the recirculating glove box antechamber overnight prior to entry.

All synthesis reactions proceeded in quartz ampoules. The ampoules were prepared from quartz tubes (19 mm O.D., 17 mm I.D., GM Associates, Oakland, CA and Technical Glass Products, Painesville, $\mathrm{OH}$ ) by sealing one end in a propane-oxygen flame and cutting to $45 \mathrm{~cm}$ lengths. Prior to use, the ampoules were submerged in a $10 \mathrm{wt} \%$ solution

of $\mathrm{KOH}_{(\mathrm{aq})}$ for at least $48 \mathrm{~h}$, rinsed thoroughly in water, and stored in a $>100{ }^{\circ} \mathrm{C}$ oven until use. Inside the recirculating glove box, we loaded cesium bromide and titanium (IV) bromide with a $10 \%$ stoichiometric excess of $\mathrm{TiBr}_{4}$ to a total reagent mass of $3 \pm 0.3 \mathrm{~g}$. For transfer out of the recirculating glove box and onto a Schlenk line for ampoule sealing, a KF-flanged butterfly valve and a 0.75 -inch, ultra-torr-style compression fitting isolated the tube from the air ambient.

Ampoules were sealed in an in-house built tube-sealing apparatus described earier, $\mathrm{S1}$ and shown in Fig. S1. In brief, the apparatus is built around a repurposed rotary evapo- 


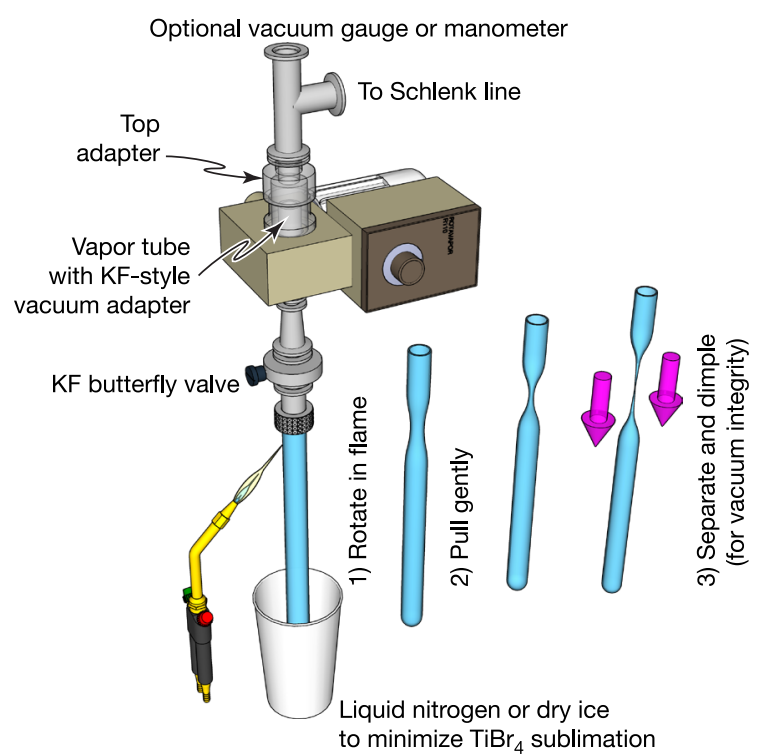

Figure S1. Cartoon depiction of the tube sealing setup. Due to the instability of $\mathrm{TiBr}_{4}$ in an air ambient, tubes are loaded in a recirculating glove box and isolated from the atmosphere with a ultratorr-to-KF-flange adapter and a KF-flange butterfly valve. A styrofoam cup holding either liquid nitrogen or dry ice minimizes sublimation of $\mathrm{TiBr}_{4}$ during tube evacuation.

rator (Rotavapor R110, BÜCHI) to rotate the tube during in vacuo sealing to assure even, consistent heating, and steady, safe flamework. In place of a solvent trap, a direct connection to the Schlenk line affords high vacuum conductivity and rapid pumping (50 mm Rotavap flange adapter, Adams \& Chittenden Scientific Glass Coop., Berkeley, CA). Sealing against the rotavapor vacuum gasket, a 6061 aluminum tube replaces the standard vapor tube and with a bottom connection consisting of a KF flange in place of a traditional 24/40 \$ fitting. For sealing, a loaded tube was transferred to the sealing apparatus and initially evacuated with the butterfly valve closed to minimize possible contamination of the highly reactive $\mathrm{TiBr}_{4}$ with oxygen. Prior to opening the butterfly valve, the reactants at the bottom of the ampoule were submerged in liquid nitrogen to minimize unwanted vacuum sublimation of $\mathrm{TiBr}_{4}$. Following evacuation of the connecting tubing, the butterfly valve was opened to the Schlenk line slowly to fully evacuate the quartz tube. The quartz tube was rotated at $\sim 1 \mathrm{~Hz}$ and a propane-oxygen flame sealed the tube to yield a 22-25-cm-long ampoule.

High-temperature melt syntheses utilized horizontally mounted 5-cm-I.D. tube furnaces that were constructed from two abutting tubular ceramic heaters (CRFC-series, Omega Engineering, Norwalk, CT). A 30-cm-long and a 15-cm-long heater defined two independently controlled heating zones. Temperature controllers (Love 32B, Dwyer Instruments, Michigan City, IN) recorded temperatures at K-type thermocouples (Nextel ceramic braid insulation, Omega Engineering, spot welded in house) that were positioned at the center of each zone. An in-house-written LabVIEW program interfaced with the temperature controllers for conducting temperature ramp-and-soak profiles and recording temperature data. 
Melt reactions utilized a 15-hour linear ramp step to $680^{\circ} \mathrm{C}$ at the reactant end of the ampoule and $710{ }^{\circ} \mathrm{C}$ at the opposite end of the ampoule, and a 25-hour soak step at 680 and $710^{\circ} \mathrm{C}$. Cooling consisted of a 96-hour linear ramp step to room temperature during which the reactant end remained $30{ }^{\circ} \mathrm{C}$ lower in temperature relative to the opposite end of the ampoule. Certain synthesis runs utilized a rapid cooldown of the opposite end of the ampoule starting at $300^{\circ} \mathrm{C}$ in the cooldown phase so as to getter unreacted $\mathrm{TiBr}_{4}$ away from the reactant end, but that did not yield large-grain material and we do not consider that cooldown strategy further. Following synthesis, ampoules were transferred to a flush box and cracked open to reveal dark-red, polycrystalline $\mathrm{Cs}_{2} \mathrm{TiBr}_{6}$ samples of $\sim 1 \mathrm{~cm}^{3}$ total volume with visible facets in excess of $1 \mathrm{~mm}^{2}$ in area. Crystals were transferred from the flush box to the recirculating glove box in sealed containers for storage until further use.

\section{S1.3. Surface Processing}

Samples analyzed via photoelectron spectroscopy included nascent crystals with no surface preparation, DCM-rinsed, physically abraded, a sequential rinsing in DCM followed by physical abrasion, UHV surface cleaving, and air exposure following UHV cleaving. In the flush box, rinsing consisted of repeated $\sim 1 \mathrm{~mL}$ applications of dichloromethane that yielded a yellow coloration of the collected liquid that likely resulted from the removal of interfacial $\mathrm{TiBr}_{4}$. Rinsing was repeated with fresh DCM until the collected liquid remained colorless. Physical abrasion in the flush box consisted of sanding with 400grit sandpaper (Saint-Gobain Abrasives Inc., Worcester, MA). Typical durations between physiochemical processing, sample mounting and air-free sample transfer to the XPS, and subsequent evacuation of the sample transfer suitcase was 10-20 minutes.

A modification to the photoelectron spectrometer described below enabled UHV surface cleaving. We fastened a commercial straight-edge razor blade to the magnetically coupled transport arm that moves samples between the ultrahigh vacuum analysis chamber and the high-vacuum, temperature-programmed-desorption side chamber. ${ }^{\mathrm{S} 2}$ The fastened blade enabled cleaving of a $\mathrm{Cs}_{2} \mathrm{TiBr}_{6}$ sample mounted on a PHI sample puck (Model 190; Physical Electronics, Inc.; via RBD Instruments, Inc., Bend, OR) while in the analysis chamber. During cleaving, analysis chamber pressures remained at or below $1 \times 10^{-8}$ torr as measured at a UHV ion gauge. XPS and UPS analyses directly followed UHV cleaving on freshly exposed regions of the sample with a maximum of 20 minutes between sample cleaving and photoelectron data acquisition.

For air exposures that followed UHV cleaving and photoelectron analyses, samples were transferred from the analysis chamber to the instrument load lock that was subsequently equilibrated to an air ambient. Following ten minutes under the ambient at- 
mosphere of $20{ }^{\circ} \mathrm{C}$ and $40-50 \%$ relative humidity, the load lock was re-evacuated for photoelectron analyses.

\section{S1.4. Photoelectron Spectroscopy}

A PHI 5600 multitechnique system with a third-party data acquisition system (RBD Instruments, Inc.) acquired all X-ray and UV photoelectron spectra detailed earlier. ${ }^{\text {S1-4 }}$ Analysis chamber base pressures were $<1 \times 10^{-9}$ Torr. A hemispherical energy analyzer collected the photoelectrons for both X-ray and ultraviolet photoelectrons. A monochromated $\mathrm{Al} \mathrm{K} \alpha$ source provided X-rays at a $90^{\circ}$ angle with respect the takeoff angle for the energy analyzer. Typical XPS data collection utilizes a level sample puck that yields $45^{\circ}$ angles both for the incoming monochromated X-radiation and for the photoelectron takeoff angle with respect to the sample normal angle. Typical UPS data collection utilizes direct alignment of the sample normal angle with the photoelectron takeoff angle that yields a $\sim 51^{\circ}$ angle between that angle and the incoming He I UV radiation.

In the recirculating glove box, samples were mounted on freshly cleaned sample pucks using carbon adhesive tape. For samples to be subjected to UHV cleaving, we additionally applied Hysol 1C epoxy between the sample and the puck for rigidity and allowed the epoxy to cure for $48 \mathrm{~h}$. Epoxy components were pumped in small volumes with sufficient time in the antechamber to effect degassing. A vacuum suitcase (Transfer Engineering and Manufacturing, Inc., Fremont, CA) enabled air-free transfer of puck-mounted $\mathrm{Cs}_{2} \mathrm{TiBr}_{6}$ samples from the recirculating glove box to the instrument load lock.

$\mathrm{X}$-ray photoelectron acquisitions included wide-energy survey scans as well as highresolution scans of the $\mathrm{C} 1 \mathrm{~s}, \mathrm{O} 1 \mathrm{~s}, \mathrm{Ti} 2 \mathrm{p}, \mathrm{Cs} 3 \mathrm{~d}_{5 / 2}$, and $\mathrm{Br} 3 \mathrm{~d}$ regions for all samples. Survey spectra utilized a $117 \mathrm{eV}$ analyzer pass energy, a $0.5 \mathrm{eV}$ step size, and a 50-ms-per-step dwell time. High-resolution spectra employed a $23.5 \mathrm{eV}$ pass energy, a $0.025 \mathrm{eV}$ step size, and a 50-ms-dwell-time per step. Nascent samples with no surface processing necessitated charge neutralization, while all other samples did not require charge neutralization. Neutralization parameters were optimized to yield a $C 1$ s feature from adventitious contaminants of minimal full-width-at-half maximum (fwhm) value that was corrected to $285.0 \mathrm{eV}$ during post-acquisition analyses.

Peak quantification utilized an in-house-developed LabVIEW program based on published spectral shapes and corrected for instrument-specific sensitivity factors, ${ }^{55-6}$ and background-energy-loss functions. ${ }^{S 7-9} \mathrm{~A}$ universal Tougaard-style baseline with with $B=$ $2900 \mathrm{eV}^{2}$ and $C=1643 \mathrm{eV}^{2}$ was utilized for the $\mathrm{Cs} 3 \mathrm{~d}_{5 / 2}$ and $\mathrm{Br} 3 \mathrm{~d}$ regions; ${ }^{\mathrm{S}-8}$ a Shirleystyle baseline was used for the Ti $2 p$ region; ${ }^{S 9}$ and linear baselines described the $C 1$ s, and $\mathrm{O} 1$ s regions. Data fitting employed baseline-corrected, pseudo-Voight, GL $(x)$-style 
functions where $x$ non-linearly scales from a pure Gaussian $(x=0)$ to a pure Lorentzian $(x=100) .{ }^{\mathrm{S} 6} \mathrm{GL}(30)$ peak shapes described the $\mathrm{C} 1 \mathrm{~s}, \mathrm{O} 1 \mathrm{~s}, \mathrm{Br} 3 \mathrm{~d}$, and Ti $2 \mathrm{p}_{1 / 2}$ regions. GL(70) peak shapes were utilized for the Cs $3 d_{5 / 2}$ and Ti $2 p_{3 / 2}$ regions. Fits that employ multiple peaks within a spectral region were constrained to identical full-width-at-halfmaximum (fwmh) values for each peak, with the exception of Ti $2 p$ spectra. We report sensitivity-factor-corrected XPS peak areas (raw peak area $\div$ respective sensitivity factor). We employed instrument- and analyzer-specific sensitivity factors for photoelectron collection at $90^{\circ}$ with respect to the incident $X$-ray beam. Sensitivity factors for Cs $3 \mathrm{~d}_{5 / 2}$, $\mathrm{C} 1 \mathrm{~s}, \mathrm{O} 1 \mathrm{~s}, \mathrm{Br} 3 \mathrm{~d}$, and Ti $2 \mathrm{p}$ were $6.032,0.296,0.711,0.895$, and 1.789 respectively. ${ }^{\mathrm{S} 5}$

For ultraviolet photoelectron spectroscopy, a gas discharge lamp (UVS 40A2, Prevac, Rogów, Poland) generated the He I spectroscopic line, $\mathbf{E}_{\mathrm{HeI}}=h v=21.218 \mathrm{eV}$. We utilize a $-35 \mathrm{~V}$ substrate bias versus the grounded sample chamber to both maximize the desired spectral contributions of electrons from the sample and isolate unwanted secondary electrons from the analyzer. We chose the $-35 \mathrm{~V}$ bias based on an invariance in spectral features and good linearity in analyzer response for UP spectra of a freshly $\mathrm{Ar}^{+}$-sputtered gold foil between biases of -20 and $-50 \mathrm{~V}$ vs the sample chamber ground. UP spectra of a freshly $\mathrm{Ar}^{+}$sputtered gold foil verified instrumental calibration with the bias-corrected Fermi level of gold, $\mathrm{E}_{\mathrm{F}, \mathrm{Au}} \equiv 0.00 \pm 0.05 \mathrm{eV}$. From plots of photoelectron counts vs biascorrected binding energy, the $\mathrm{x}$-axis intercept of linear regressions quantifies a secondaryelectron-cutoff energy, $\mathbf{E}_{\mathrm{SEC}}$, and a valence-band-edge (maximum) energy, $\mathbf{E}_{\mathrm{VBM}}$. Considering the binding energy calibration of the instrument, we report sample work function values, $\phi$, as the difference between $\mathbf{E}_{\mathrm{He}}$ and the secondary electron cutoff energy, or $\phi$ $=\mathbf{E}_{\mathrm{HeI}}-\mathbf{E}_{\mathrm{SEC}}$. We report Fermi-level energies vs valence-band-maximum energies, or $\mathbf{E}_{\mathrm{F}}-\mathbf{E}_{\mathrm{VBM}}$. As ultrahigh vacuum bakeouts can yield a small perturbation in instrument calibration and spatial alignment that would deleteriously affect data analyses, both gold calibration and $\mathrm{Cs}_{2} \mathrm{TiBr}_{6}$ data were acquired during the same "bakeout window".

\section{S1.5. X-ray Diffraction}

A Bruker-AXS D8 focus powder X-ray diffractometer with $\mathrm{Cu} \mathrm{K} \alpha$ radiation collected Xray diffraction $(\mathrm{XRD})$ data in the range of $10-45^{\circ}(2 \theta)$ with a step size of $0.05^{\circ}$ and a 5-s-dwell-times per step. X-ray tube operating conditions were $40 \mathrm{kV}$ and $40 \mathrm{~mA}$. In the flush box, $\mathrm{Cs}_{2} \mathrm{TiBr}_{6}$ samples were sandwiched between the XRD sample mount and lowstatic Kapton tape (KaptonTape.com, Torrance, CA) to minimize contact with ambient air during data acquisition. For studies of the evolution of XRD trace features as a function of air exposure, the Kapton tape was removed for sample exposure to an air ambient. Air exposure utilized two distinct protocols. In one protocol, either 10 or 100 minute 
air exposures were followed by transfer back into the recirculating glove box, resealing under Kapton, and full 10-45 trace acquisitions as above. In the second protocol, Kapton tape was removed and a sample was successively and repetitively scanned in 10-minute intervals at $2 \theta$ angles between of $28-30.5^{\circ}(2 \theta)$ with a step size of $0.02^{\circ}$ and 4 -s dwell times per step. The $\mathrm{Cs}_{2} \operatorname{TiBr}_{6}(222)$ and $\mathrm{CsBr}(110)$ reflections in the XRD traces gathered in the range of $28-30.5^{\circ}(2 \theta)$ were fitted in Igor Pro (WaveMetrics, Lake Oswego, OR) to yield the peak areas $A_{\mathrm{Cs}_{2} \mathrm{TiBr}_{6}}$ and $A_{\mathrm{CsBr}}$. The relative intensities were subsequently calculated by normalizing the $A_{\mathrm{Cs}_{2} \mathrm{TiBr}_{6}}$ and $A_{\mathrm{CsBr}}$ values to the total fitted peak area, $A_{\mathrm{Cs}_{2} \mathrm{TiBr}_{6}}+A_{\mathrm{CsBr}}$.

\section{S1.6. UV-Vis Spectroscopy}

A UV-Visible spectrometer (Evolution 300, Thermo Fisher Scientific, Waltham, MA) collected scattered light from $\mathrm{Cs}_{2} \mathrm{TiBr}_{6}$ samples mounted in a Praying Mantis diffuse reflection accessory (Harrick Scientific Products, Pleasantville, NY) and sealed with Kapton tape. The sample holder with Kapton tape serves as the background reference for each scan. Scans were obtained in a range of $300 \mathrm{~nm}$ to $1000 \mathrm{~nm}$, using a scan speed of 160 $\mathrm{nm} / \mathrm{min}$ and a bandwidth of 1 in absorbance mode.

\section{S1.7. Imaging}

A Tescan Analytics Vega3 (Tescan Orsay Holding, A.S., Brno-Kohoutovice, Czechia) SEM with a tungsten cathode operating at a $10 \mathrm{kV}$ acceleration potential and a secondaryelectron detector acquired the images in Fig 1B-C. A Hitachi TM3000 TableTop SEM (Hitachi High-Tech America, Inc., Schaumburg, Illinois) operating with a $15 \mathrm{kV}$ acceleration potential, a four-segment backscatter electron detector, and a silicon drift detector for energy-dispersive X-ray (EDX) analysis yielded the images in Fig. 1D-E. The EDX was calibrated using a copper grid standard and a built-in calibration curve provided by Hitachi. Samples for SEM and EDX analyses were prepared in the recirculating glovebox and transported to the instruments in sealed vials with rapid transfer for SEM analyses.

\section{S1.8. Computational Methods}

We modeled a bulk cell of $\mathrm{Cs}_{2} \mathrm{TiBr}_{6}$ using DFT to obtain details on its electronic structure. We used a unit cell based on a $F m \overline{3} m$ space group (number 225) with a unit cell length of $a=b=c=1.092 \mathrm{~nm}$ and a Ti-Br distance of $0.273 \mathrm{~nm}$ to match published XRD results. We used the Vienna Ab Initio Simulation Package (VASP) ${ }^{\mathrm{S} 10-13}$ for the simulations. Core electrons were represented by projector augmented wave (PAW) potentials, ${ }^{\text {,14-15 }}$ while valence electrons were represented by a plane-wave basis set with a cutoff energy of $450 \mathrm{eV}$. Calculations used the HSE06 exchange correlation functional, ${ }^{\mathrm{S} 16}$ and were spinpolarized. We used a $2 \times 2 \times 2 k$-point mesh to sample reciprocal space. 


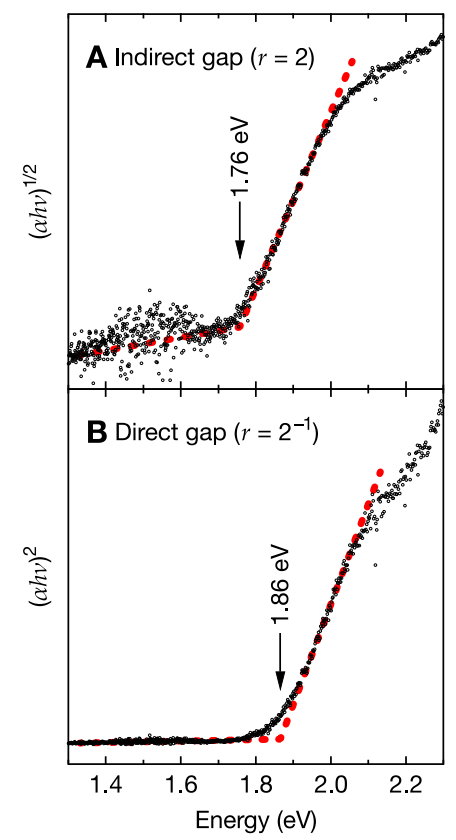

Figure S2. UV-Vis data plotted as $(\alpha h v)^{1 / r}$ vs energy for large-grain $\mathrm{Cs}_{2} \mathrm{TiBr}_{6}$ showing (A) the indirect band gap of the material at $1.76 \mathrm{eV}$ for $r=2$, and (B) the direct band gap of the material at $1.86 \mathrm{eV}$ when $r=2^{-1}$.

\section{S2. SUPPLEMENTAL RESULTS}

\section{S2.1. Absorption-Spectra-Derived Band Gap Energies}

As synthesized from a high-temperature melt and a long cooling period, crystals appeared red-black to black with red fringing along thin edges. Scattering patterns demonstrated crystal facets with areas in excess of $1 \mathrm{~mm}^{2}$.

Figure S2 presents $(\alpha h v)^{1 / r}$-vs-energy Tauc plots for large-grain $\mathrm{Cs}_{2} \mathrm{TiBr}_{6}$ samples that were loaded into the UV-Vis sample holder under an inert atmosphere and isolated with Kapton tape. Linear fits to the absorption onset feature when $r=2$ in frame A reveal a baseline-corrected energy-axis intercept of $1.76 \mathrm{eV}$, and $1.86 \mathrm{eV}$ when $r=2^{-1}$ as presented in frame B. Therefore, extrapolations respectively indicate indirect and direct forbidden energy gaps of 1.76 and $1.86 \mathrm{eV}$ for the dark-red, large-grain $\mathrm{Cs}_{2} \mathrm{TiBr}_{6}$ material under study. This agrees well with early reports of $1.8 \mathrm{eV}$ band gaps for thin film $\mathrm{Cs}_{2} \mathrm{TiBr}_{6},{ }, 17-18$ and somewhat lower as compared to the $1.9 \mathrm{eV}$ indirect and $2.0 \mathrm{eV}$ direct band gaps from recent powder studies. ${ }^{\text {S19 }}$ 


\section{References}

S1. Martin, J. L.; Stoflet, R.; Carl, A. D.; Himmelberger, K. M.; Granados-Fócil, S.; Grimm, R. L. Quantification of Surface Reactivity and Step-Selective Etching Chemistry on Single-Crystal BiOI(001). Langmuir 2020, 36, 9343-9355. https : //doi .org/10.1021/acs . langmuir. 0c00980

S2. Gao, W. R.; Zielinski, K.; Drury, B. N.; Carl, A. D.; Grimm, R. L. Elucidation of Chemical Species and Reactivity at Methylammonium Lead Iodide and Cesium Tin Bromide Perovskite Surfaces via Orthogonal Reaction Chemistry. J. Phys. Chem. C 2018, 122, 17882-17894.

https://doi.org/10.1021/acs.jpcc.8b05352

S3. Carl, A. D.; Grimm, R. L. Covalent Attachment and Characterization of Perylene Monolayers on $\mathrm{Si}(111)$ and $\mathrm{TiO}_{2}$ for Electron-Selective Carrier Transport. Langmuir 2019, 35, 9352-9363. https://doi.org/10.1021/acs. langmuir.9b00739

S4. Carl, A. D.; Kalan, R. E.; Obayemi, J. D.; Kana, M. G. Z.; Soboyejo, W. O.; Grimm, R. L. Synthesis and Characterization of Alkylamine-Functionalized Si(111) for Perovskite Adhesion With Minimal Interfacial Oxidation or Electronic Defects. ACS Appl. Mater. Interfaces 2017, 9, 34377-34388. https://doi.org/10.1021/acsami.7b07117

S5. Moulder, J. F.; Stickle, W. F.; Sobol, P. E.; Bomben, K. D. Handbook of X-ray Photoelectron Spectroscopy; Physical Electronics Division: Perkin-Elmer Corporation, 1992.

S6. Fairley, N. Peak Fitting in XPS. http://www . casaxps .com/help_manual/manual_updates/peak_ fitting_in_xps.pdf (accessed August 2015).

S7. Jansson, C.; Hansen, H. S.; Yubero, F.; Tougaard, S. Accuracy of the Tougaard Method for Quantitative Surface Analysis. Comparison of the Universal and REELS Inelastic Cross Sections. J. Electron Spectrosc. Relat. Phenom. 1992, 60, 301-319. https://doi .org/10.1016/0368-2048(92)80025-4

S8. Tougaard, S. Formalism for Quantitative Surface Analysis by Electron Spectroscopy. J. Vac. Sci. Technol., A 1990, 8, 2197-2203. https://doi.org/10.1116/1.577037

S9. Shirley, D. A. High-Resolution X-Ray Photoemission Spectrum of the Valence Bands of Gold. Phys. Rev. B 1972, 5, 4709-4714. https://doi.org/10.1103/PhysRevB.5. 4709

S10. Kresse, G.; Hafner, J. Ab initio Molecular Dynamics for Liquid Metals. Phys. Rev. B 1993, 47, 558-561. https://doi.org/10.1103/PhysRevB.47.558

S11. Kresse, G.; Hafner, J. Ab initio Molecular-Dynamics Simulation of the Liquid-Metal-AmorphousSemiconductor Transition in Germanium. Phys. Rev. B 1994, 49, 14251-14269. https://doi.org/10.1103/PhysRevB.49.14251

S12. Kresse, G.; Furthmüller, J. Efficient Iterative Schemes for Ab initio Total-Energy Calculations Using a Plane-Wave Basis Set. Phys. Rev. B 1996, 54, 11169-11186.

https://doi.org/10.1103/PhysRevB.54.11169

S13. Kresse, G.; Furthmüller, J. Efficiency of Ab-initio Total Energy Calculations for Metals and Semiconductors Using a Plane-Wave Basis Set. Comp Mater. Sci. 1996, 6, 15-50. https://doi.org/10.1016/0927-0256(96)00008-0

S14. Kresse, G.; Joubert, D. From Ultrasoft Pseudopotentials to the Projector Augmented-Wave Method. Phys. Rev. B 1999, 59, 1758-1775. https://doi.org/10.1103/PhysRevB.59.1758 
S15. Blöchl, P. E. Projector Augmented-Wave Method. Phys. Rev. B 1994, 50, 17953-17979. https://doi.org/10.1103/PhysRevB.50.17953

S16. Krukau, A. V.; Vydrov, O. A.; Izmaylov, A. F.; Scuseria, G. E. Influence of the Exchange Screening Parameter on the Performance of Screened Hybrid Functionals. J. Chem. Phys. 2006, 125, 224106. https://doi.org/10.1063/1.2404663

S17. Chen, M.; Ju, M.-G.; Carl, A. D.; Zong, Y.; Grimm, R. L.; Gu, J.; Zeng, X. C.; Zhou, Y.; Padture, N. P. Cesium Titanium(IV) Bromide Thin Films Based Stable Lead-free Perovskite Solar Cells. Joule 2018, 2, 558-570. https://doi .org/10.1016/j . joule.2018.01.009

S18. Ju, M.-G.; Chen, M.; Zhou, Y.; Garces, H. F.; Dai, J.; Ma, L.; Padture, N. P.; Zeng, X. C. EarthAbundant Nontoxic Titanium(IV)-based Vacancy-Ordered Double Perovskite Halides with Tunable 1.0 to 1.8 eV Bandgaps for Photovoltaic Applications. ACS Energy Lett. 2018, 3, 297304. https://doi.org/10.1021/acsenergylett.7b01167

S19. Euvrard, J.; Wang, X.; Li, T.; Yan, Y.; Mitzi, D. B. Is $\mathrm{Cs}_{2} \mathrm{TiBr}_{6}$ a Promising Pb-free Perovskite for Solar Energy Applications? J. Mater. Chem. A 2020, 8, 4049-4054.

https://doi.org/10.1039/C9TA13870F 